\title{
Emotional expression affects the accuracy of gaze perception
}

\author{
Janek S. Lobmaier • Matthias Hartmann • \\ Andreas J. Volz • Fred W. Mast
}

Published online: 4 May 2012

(C) Springer Science+Business Media, LLC 2012

\begin{abstract}
Emotional facial expressions are powerful social cues. Here we investigated how emotional expression affects the interpretation of eye gaze direction. Fiftytwo observers judged where faces were looking by moving a slider on a measuring bar to the respective position. The faces displayed either an angry, happy, fearful or a neutral expression and were looking either straight at the observer, or were rotated $2^{\circ}, 4^{\circ}, 6^{\circ}$ or $8^{\circ}$ to the left and right. We found that happy faces were interpreted as directed closer to the observer, while fearful and angry faces were interpreted as directed further away. Judgments were most accurate for neutral faces, followed by happy, angry and fearful faces. These findings are discussed on the background of the "self-referential positivity bias", suggesting that happy faces are preferably interpreted as directed towards the self while negative emotions are interpreted as directed further away.
\end{abstract}

Keywords Gaze perception - Full gaze awareness · Emotion · Positivity bias

\section{Introduction}

Understanding another individual's intentions is essential for everyday social interactions. Humans use various cues to predict future behavior of their social interaction

J. S. Lobmaier $(\varangle) \cdot$ M. Hartmann · A. J. Volz · F. W. Mast Department of Psychology, University of Bern, Muesmattstrasse 45, 3012 Bern, Switzerland

e-mail: janek.lobmaier@psy.unibe.ch

J. S. Lobmaier · M. Hartmann · F. W. Mast Center for Cognition, Learning, and Memory, University of Bern, Bern, Switzerland partners. One important cue is direction of eye gaze. Where somebody is looking tells us where his or her focus of attention is. Gale and Monk (2000) distinguished between full, partial and mutual gaze awareness. Full gaze awareness is the knowledge of the precise location in the environment where a person is looking, while partial-gaze awareness describes the knowledge about the general direction of the eye gaze. Finally, mutual gaze awareness describes the capability to detect whether a person is making eye contact or not. Accuracy of gaze perception varies across the three different types of gaze awareness. Studies have shown that humans are very accurate regarding mutual and partial eye gaze awareness while being slightly less accurate for full gaze awareness (Lee et al. 1998; Lobmaier et al. 2006; Schwaninger et al. 2005; Symons et al. 2004). Our capability to interpret eye gaze depends on different sources of information. One important source is the iris/sclera ratio, which is used to compute the direction of regard (Ando 2002). Also, the posture of the looker's head (Langton 2000) and the presence of objects in the attended space influence the interpretation of gaze direction (Lobmaier et al. 2006).

Emotional expression is yet another important facial cue to social interactions. From their facial expression we can infer the motivational state of a counterpart. An angry face might pose a threat, while a happy face denotes a benevolent attitude and may give rise to positive social interactions. Together with the direction of eye gaze the emotional expression indicates whether the expressed emotion is indeed directed at me or elsewhere. In fact, recent findings have suggested that gaze direction and facial expression are combined in order to process emotionally relevant facial information: direct gaze enhances the perception of approach-oriented emotions such as anger or joy, while averted eye gaze enhances the perception of 
avoidance-oriented emotions such as sadness and fear (Adams and Kleck 2003, 2005). Sander and colleagues (Sander et al. 2007) extended these findings by using dynamic stimuli. They artificially created stimuli that showed faces turning from a neutral to a happy, angry or fearful expression. After seeing each stimulus, participants were asked to rate the intensity as well as how strongly each of the six basic emotions (fear, anger, disgust, happiness, surprise, sadness) was represented in the respective expression. Thus, participants were requested to use continuous expression scales allowing them not only to discriminate a given expression in the context of multiple labels, but also to state which expressions were present in the face. The results revealed that the emotions were generally correctly recognized above chance level, but that gaze direction influenced the perceived intensity of fearful and angry expressions. Angry faces were rated as more angry when gaze was direct, while fearful expressions appeared more fearful when gaze was averted. But in contrast to the findings of Adams and Kleck (2003, 2005), Sander et al. (2007) found that perception of happiness was unaffected by gaze direction. They interpreted their findings in favour of the appraisal theory of emotion processing, such that gaze direction modulates the relevance of the emotion.

Conversely, the emotional expression modulates the perceived gaze direction. In a recent study we found that happy faces were more likely to be perceived as looking at the observer than were angry, fearful and neutral faces (Lobmaier and Perrett 2011; Lobmaier et al. 2008). We interpreted this finding as a "self-referential-positivity bias", suggesting that people prefer to think that somebody else's happiness is directed to oneself, whereas negative emotions are rather perceived as directed elsewhere. Such a self-referential bias suggests that people generally have positive self-concepts which may be healthy for selfesteem.

Most previous studies investigating the influence of emotional expression on gaze perception focused on mutual eye gaze awareness (Adams and Franklin 2009; Lobmaier and Perrett 2011; Lobmaier et al. 2008; Rimmele and Lobmaier 2011). For example, in the study of Adams and Franklin (2009) participants were asked to decide as quickly as possible whether a presented face was looking at them or not. They found that averted gaze was processed more quickly and accurately when coupled with fear and direct gaze was processed faster and more accurately when the stimulus face showed an angry expression. Other researchers have used a gaze-cueing paradigm with emotional faces as stimuli (e.g., Fox et al. 2007; Putman et al. 2006; Tipples 2006). In such spatial orienting tasks participants typically are presented with a centrally located cue face with the gaze either directed to the left or to the right. After a short interval, a target appears peripherally, either on the left or right side of the face, to which participants have to react as quickly as possible. Reaction times are consistently shorter, if gaze direction and target location are congruent (e.g., if the gaze is directed to the left and target appears on the left side) compared to when gaze direction and target location are incongruent (e.g., gaze to left, target on the right). A number of studies have shown that this gaze cueing effect is modulated by the emotional expression on the cue face. For example, a cue face showing a fearful expression has been shown to potentiate the gaze cueing effect (Putman et al. 2006; Tipples 2006). However, note that some authors have failed to find an influence of the emotional expression on the gaze cueing effect (e.g., Hietanen and Leppanen 2003).

While most findings suggest that the processing of emotional expression and gaze direction mutually interact, no study to our best knowledge has been conducted investigating whether emotional expression modulates full gaze awareness. Mutual gaze awareness is pertinent in dyadic settings, where we have to detect whether the other person is making eye contact or not. Full gaze awareness is characterized by a triadic setting: we have to identify the exact location in space where another person is looking. Thus, mutual gaze awareness focuses on regulating direct social interaction whereas full gaze awareness goes beyond dyadic interactions. Full gaze awareness can be used to reveal an individual's focus of attention, point of reference and intentions (Symons et al. 2004) and thus calls for a much more sophisticated processing mechanism. A fearful face looking at a certain point in the environment might inform us of a potential threat and might draw our attention to sources of danger. Thus, the ability to tell where exactly in the environment another person is looking might help to avoid harm. It is therefore likely that the kind of expression will influence the accuracy to detect the direction of eye gaze in a triadic setting. Thus, this study aims at extending previous findings to a triadic setting, where observers are asked to indicate where exactly a looker is directing his or her gaze. Knowing where in the environment somebody is looking has a different significance than knowing whether somebody is making eye contact or not. Whereas the latter is immediately relevant for the self, gaze that is directed somewhere in the environment may be more relevant for others. Thus, it is unclear whether the self-referential positivity bias will also survive a change of task (i.e., asking people where in the environment somebody is looking instead of asking whether somebody is making eye contact or not).

The aim of this study was to investigate whether the emotional expression influences the perceived direction in which somebody is looking. We note that eye gaze is not the only body cue that provides information on the 
direction of a person's attention. Head orientation for example also provides information about where someone is attending (Perrett et al. 1992). Knowing where somebody is looking is thus not dependent on a simple detection of eye position but rather involves the integrated perception of eye and head position (Langton 2000; Langton et al. 2000). In this paper we refrain from differentiating between gaze and head direction and use the word "gaze" to describe the direction in which somebody is looking (following Lobmaier and Perrett 2011; Lobmaier et al. 2008; Rimmele and Lobmaier 2011). Ultimately, such a definition of gaze direction better accounts for the fact that when communicating an emotional state to somebody, we will most certainly also direct our facial expression towards this person. Thus it seems ecologically more valid to use stimuli where head and eye gaze point in the same direction.

Following previous findings, we expect the emotional expression to modulate the perceived gaze direction. Specifically, we predict the gaze of happy faces to be perceived as shifted towards the observer, compared to the gaze of fearful, angry and neutral faces. Such a result would be in line with our previous findings, showing that happy faces are preferably associated with the self, whereas angry and fearful faces are rather interpreted as directed away from ourselves. In terms of accuracy, we expect participants to determine the gaze direction of neutral faces most correctly, as in neutral faces no emotional expression interferes with the processing gaze direction.

\section{Methods}

\section{Participants}

Fifty-two (26 male, 26 female) undergraduate students from the University of Bern participated in the study (mean age 24.2, ranging from 19 to 43). Participants received academic credit points in return for their participation. All participants reported normal or corrected to normal visual acuity and gave written informed consent to take part in this study.

\section{Apparatus}

The study was run on a PC using SuperLab 4.0. The stimuli were presented on a $21^{\prime \prime}$ computer screen (Samsung SyncMaster 213T) with a resolution of $1,280 \times 1,024$ pixels and a color depth of 24 bits. The participants were seated on a height-adjustable chair at a distance of $150 \mathrm{~cm}$ away from the screen. The stimulus faces appeared in the center of the computer screen with a width of approximately $15 \mathrm{~cm}$, subtending to a visual angle of approximately $5.72^{\circ}$ horizontally. A headrest ensured that viewing distance was kept constant. A custom made device consisting of an aluminium bar on which a slider was attached was positioned horizontally, $30 \mathrm{~cm}$ in front of the participant. Observers judged where the stimulus face was looking by marking the location (target point) on the aluminium bar. Specifically, participants moved the slider on this bar to the exact position where they perceived the extension of the gaze line of the face to intersect with the bar (cf. Lobmaier et al. 2010). On the rear side of the bar a tape measure was attached, which was only visible for the experimenter. Judgments were recorded by registering the positions of the slider.

\section{Stimuli}

Three-dimensional images were acquired using a $3 \mathrm{dMD}$ (www.3dMd.com) surface capture system that uses unstructured light (a speckle pattern) to perform stereo matching of two pairs of images (one for each of the left and right side of the face). The system also collects a registered color texture map from each side of the face, and combines the left and right sides into a single triangular mesh data structure. Faces of six actors ( 3 male, 3 female) were captured while expressing one of four emotions (neutral, happy, fearful, and angry) and at the same time fixating their gaze on a pre-defined target point which was situated approximately $80 \mathrm{~cm}$ away from the actor, on a straight line in front of the actors. Nose, target point, and virtual camera lay on the same axis, resulting in aligned gaze and head direction. Custom-made software enabled us to freely rotate each of the six $3 \mathrm{~d}$ models. For each actor and emotional expression we extracted nine rotations in the horizontal plane $\left(-8^{\circ},-6^{\circ},-4^{\circ},-2^{\circ}, 0^{\circ}, 2^{\circ}, 4^{\circ}, 6^{\circ}\right.$, and $\left.8^{\circ}\right)^{1}$ and converted these to jpeg format (see Fig. 1 for an example). The resulting 216 images were pseudo-randomly put in a sequence according to the following rule: a face that was rotated to one side (e.g., left) was followed by a face that was rotated to the opposite side (e.g., right), or $0^{\circ}$. With this constraint, a difference in gaze direction should be noticeable for every subsequent stimulus face. A total of two sequences (an initial sequence or its reversed order) were created. Facial expressions are often asymmetrical, such that the left side of the face is more emotionally expressive and more often connotes negative emotions than the right side (Borod et al. 1997; Darwin 1872; Powell and Schirillo 2009). To avoid possible effects of facial asymmetries on gaze judgments, a second set of stimuli was created by mirroring all 216 images along the vertical axis. Sequence

\footnotetext{
${ }^{1}$ The $0^{\circ}$ position was established in an interactive pre-test, where 12 participants were asked to adjust each $3 \mathrm{~d}$ face so that it was making eye contact. Separately for each face we then averaged the adjusted angles to receive the $0^{\circ}$ angle. From these resulting in $0^{\circ}$ angles the remaining 8 gaze angles were calculated.
} 

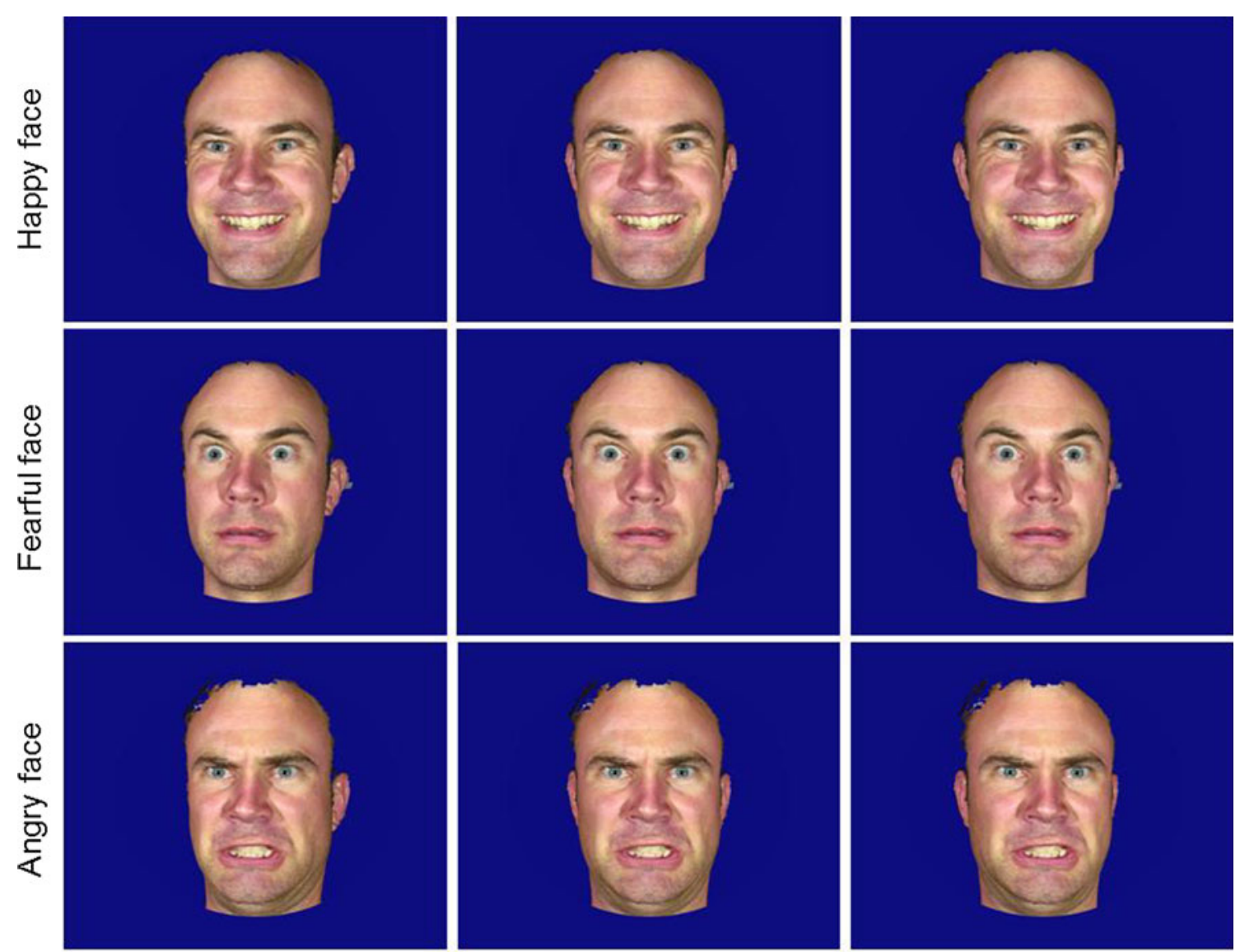

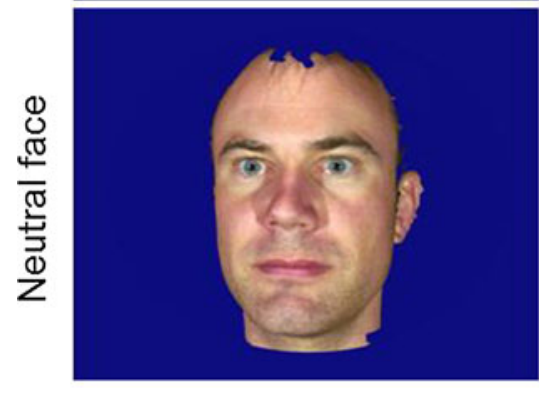

$8^{\circ}$ to the left

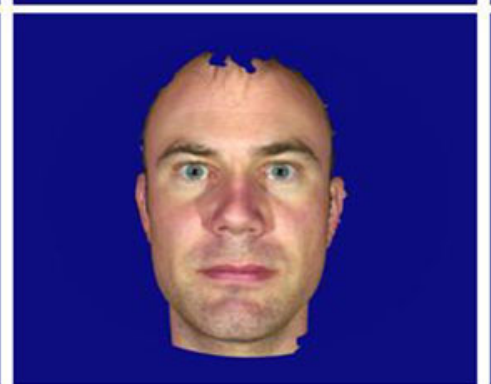

$0^{\circ}$

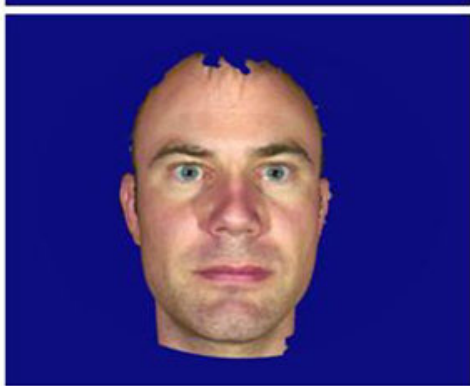

$4^{\circ}$ to the right

Fig. 1 Stimuli samples. Three different views ( $8^{\circ}$ to the left, direct and $4^{\circ}$ to the right) of a happy, fearful, angry, and neutral face

and image set (original or mirrored) were counterbalanced across participants. Prior to the main experiment, the emotional expressions were classified by 12 independent observers in a four-alternative forced-choice task. Participants had to decide which of the four emotions the face was most likely expressing (happy, angry, neutral, and fearful). The emotions were correctly recognized by more than $85 \%$ of observers (chance $=25 \%$ ).

\section{Task and procedure}

Participants were seated behind the measuring bar with their heads approximately $1.5 \mathrm{~m}$ away from the screen.
Each face was presented for $500 \mathrm{~ms}$, followed by a screen that instructed participants to indicate the perceived gaze direction on the bar by moving the slider to the respective position with their right hand. The position of the slider was noted by the experimenter according to the digits on the rear side of the bar. No feedback was given to the participant. To proceed to the next trial participants were asked to press the space bar on a keyboard in front of them.

Data analysis

The positions of the slider on the measuring bar were converted into degrees of visual angle respective to the 
straight-ahead angle of the stimulus. Specifically, we calculated the visual angle by employing following formula: $\dot{\alpha}=\operatorname{arctang}(a / b)$, whereby $a$ signifies the metric distance on the slider and $b$ equals the distance between the stimulus face and the slider. The perceived rotation angles were averaged for each rotation angle, emotional expression and participant. First, the influence of the emotional expression on perceived gaze direction was analyzed with a repeated measure ANOVA with emotional expression (angry, fearful, happy, neutral) and rotation angle (9 angles) as within factors. To test our prediction that the gaze of happy faces is perceived as shifted towards the observer, we computed three planned contrasts (paired $t$ tests) between the perceived gaze direction of happy faces and all three other emotional expressions. Secondly, we analyzed the effect of emotional expression on the accuracy of perceived gaze direction. Accuracy was calculated by dividing the perceived rotation angle by the veridical rotation angle of the stimulus face. Thus, a value of 1 denotes perfectly accurate perception of gaze direction; values below 1 imply underestimation and values larger than 1 imply overestimation of rotation angle. The influence of the emotional expression on accuracy of perceived gaze direction was analyzed by means of a repeated measure ANOVA with emotional expression (angry, fearful, happy, neutral) and rotation angle ( 9 angles) as within factors. To test our prediction that the gaze direction of neutral faces is perceived most correctly, we computed three planned contrasts (paired $t$ tests) between the accuracy of neutral faces and all three other emotional expressions. Moreover, the mean accuracy for all emotional expressions was compared to perfect accuracy (simple $t$ tests against 1).

\section{Results}

Perceived rotation angle

Repeated measures $\mathrm{ANOVA}^{2}$ with the factors emotional expression (angry, fearful, happy, neutral) and rotation angle $\left(-8^{\circ},-6^{\circ},-4^{\circ},-2^{\circ}, 0^{\circ}, 2^{\circ}, 4^{\circ}, 6^{\circ}\right.$, or $\left.8^{\circ}\right)$ revealed significant main effects of emotional expression, $F(3$, $153)=36.59, p<.001, \eta_{p}^{2}=0.42$, and of rotation angle, $F(2.24,114.46)=199.58, \quad p<.001, \quad \eta_{p}^{2}=0.80$. The interaction between emotional expression and rotation angle was significant, $F(9.12,465.25)=2.66, p=.005$, $\eta_{p}^{2}=0.05$.

Contrast analyses revealed that the gaze direction of happy faces was perceived nearer towards the self $(\mathrm{M}=4.40, \mathrm{SE}=0.27)$ as compared to neutral $(\mathrm{M}=4.85$;

\footnotetext{
2 The Huynh-Feldt epsilon correction for heterogeneity of covariances was used when sphericity could not be assumed.
}

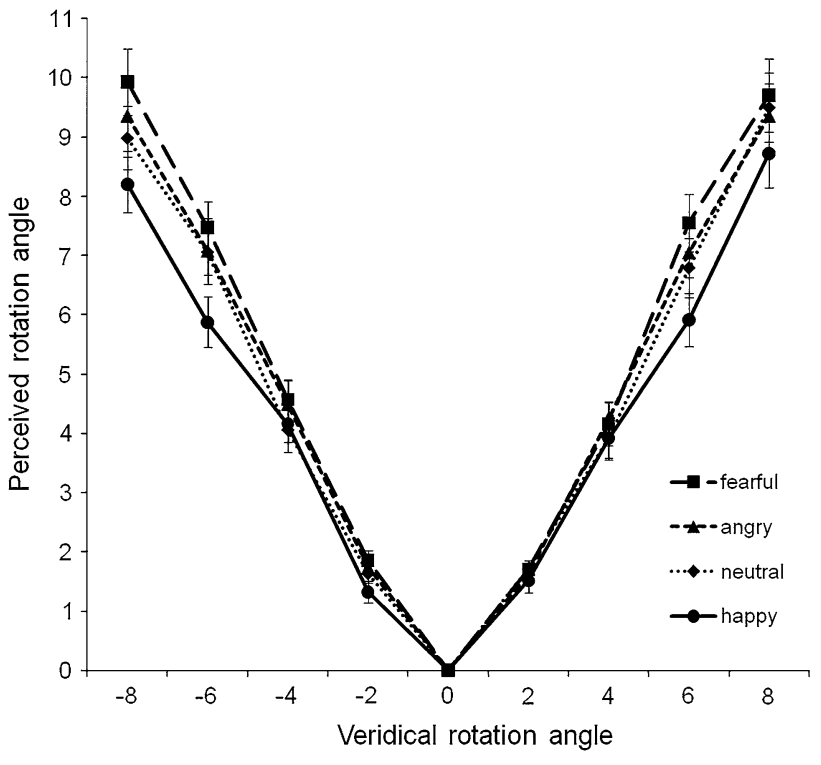

Fig. 2 Mean perceived rotation angle for each emotional expression and veridical rotation angle. Error bars depict $\pm 1 \mathrm{SE}$

$\mathrm{SE}=0.26)$, fearful $(\mathrm{M}=5.22, \mathrm{SE}=0.29)$, and angry $(\mathrm{M}=4.99, \mathrm{SE}=0.28)$ faces (all $p$ 's $<.001)$. Additional post hoc tests (Bonferroni corrected) revealed significant differences between angry and fearful $(p=.01)$, and between fearful and neutral $(p<.001)$. The main effect of rotation angle is not surprising as it reflects an increase of perceived gaze angles as head and gaze turned away from the observer.

The interaction between emotional expression and rotation angle shows that the emotional expression primarily affected the perceived rotation angle of faces that were looking further away from the self (see Fig. 2). The differential effect of emotional expression on perceived gaze direction was particularly apparent in the rotation angles $\pm 6^{\circ}$ and $8^{\circ}$.

Accuracy of perceived gaze direction

A repeated measures ANOVA with the factors emotional expression (angry, fearful, happy, neutral) and rotation angle $\left(-8^{\circ},-6^{\circ},-4^{\circ},-2^{\circ}, 0^{\circ}, 2^{\circ}, 4^{\circ}, 6^{\circ}\right.$, and $\left.8^{\circ}\right)$ revealed a significant main effect of emotional expression, $F(3$, $153)=24.14, p<.001, \eta_{p}^{2}=0.32$, as well as of rotation angle, $F(4.16,212.23)=10.98, p<.001, \eta_{p}^{2}=0.18$. The interaction between emotion and rotation angle was not significant, $F(7.29,371.88)=1.12, p=.35, \eta_{p}^{2}=0.02$.

The main effect of emotional expression indicates that emotional expressions have a differential effect on the accuracy of gaze perception. Contrast analyses revealed a difference in accuracy of gaze perception between neutral faces when compared to fearful and happy faces (both $p<.001)$ but not when compared to angry faces $(p=.13)$. 


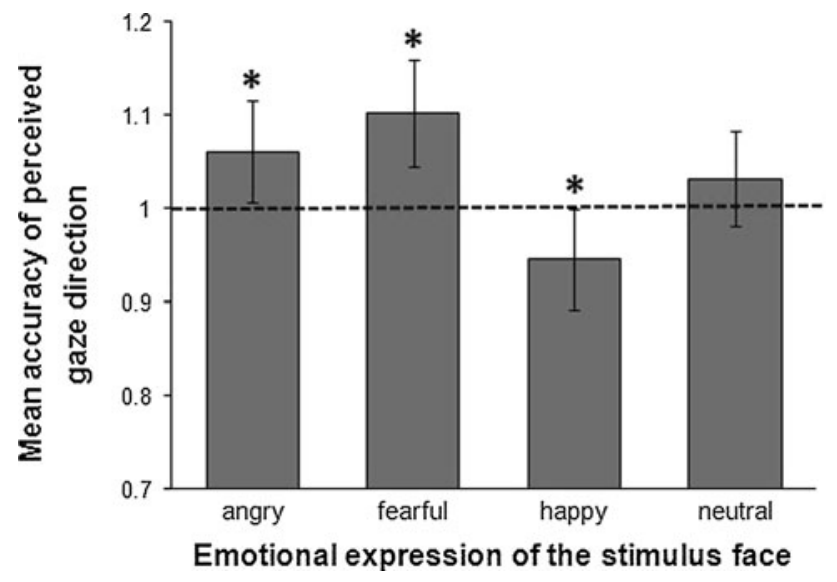

Fig. 3 Mean accuracy of perceived gaze direction for each emotional expression. Asterisks indicate significant difference from accurate perception of gaze direction represented by the dotted horizontal line. Error bars depict $\pm 1 \mathrm{SE}$

Table 1 Accuracy of perceived gaze direction for each rotation angle

\begin{tabular}{lllrc}
\hline Rotation angle $\left({ }^{\circ}\right)$ & $\mathrm{M}$ & $\mathrm{SE}$ & $t(51)$ & \multicolumn{1}{c}{$p$} \\
\hline-8 & 1.14 & 0.06 & 2.20 & .03 \\
-6 & 1.15 & 0.07 & 2.12 & .04 \\
-4 & 1.08 & 0.08 & 1.05 & .30 \\
-2 & 0.82 & 0.07 & -2.58 & .01 \\
2 & 0.82 & 0.06 & -2.94 & .005 \\
4 & 1.02 & 0.07 & 0.22 & .83 \\
6 & 1.14 & 0.07 & 1.97 & .06 \\
8 & 1.16 & 0.07 & 2.39 & .02 \\
\hline
\end{tabular}

Accuracy values below 1 imply underestimation and values above 1 overestimation of perceived rotation angle. $p<.05$ indicates that the over- or under-estimation significantly differs from accurate perception of gaze direction (as revealed by one-sample $t$ tests)

Most importantly, only neutral faces did not differ significantly from perfect accuracy, $p=.21$, confirming our prediction that neutral expressions lead to the most accurate perception of gaze direction (see Fig. 3). When the face expressed a happy emotion, gaze direction was underestimated $(p=.04)$ whereas gaze direction of angry $(p=.02)$ and fearful $(p<.001)$ faces was overestimated.

The accuracy of perceived gaze direction was also influenced by the rotation angle, irrespective of emotional expression (as indicated by the main effect of rotation angle). The gaze direction of faces that were rotated by $\pm 2^{\circ}$ was underestimated, whereas gaze direction of larger rotation angles was overestimated (Table 1).

\section{Discussion}

In this study we investigated whether the emotional expression on somebody's face affects the perception of the exact position in space where this person is looking. We found that gaze direction of happy faces was perceived as shifted towards the observer, while it was perceived as shifted away from the observer when it was accompanied by a fearful or angry expression. Gaze direction was most accurately interpreted when the expression on the lookers face was neutral, followed by angry, happy and fearful expressions.

A growing body of literature has already demonstrated that of emotional expression affects the processing of eye gaze direction (e.g., Adams and Franklin 2009; Lobmaier and Perrett 2011; Lobmaier et al. 2008; Putman et al. 2006; Tipples 2006). While some studies have found that a fearful cue face facilitates the detection of a target in a gaze cueing paradigm (Putman et al. 2006; Tipples 2006), thus demonstrating that emotional expression modulates automatic spatial orienting, others have more directly investigated the influence of emotional expression on perception of gaze direction. For example, Adams and Franklin (2009) reported shorter reaction times for detecting averted gaze when coupled with fear and for direct gaze when coupled with anger. Looking at a wider range of emotional expressions and gaze angles, we recently found that gaze was generally more likely to be interpreted as looking towards the observer if coupled with a happy compared to angry, fearful or neutral expression (e.g., Lobmaier and Perrett 2011). Extending these findings we now show that emotional expression not only modulates the feeling of being looked at, but that emotional expression also modulates how accurately the gaze target can be determined when it is located somewhere in the periphery. To our best of knowledge, we are the first to systematically investigate full gaze awareness using stimulus faces showing neutral, happy, angry and fearful expressions.

Previous studies have put forward the shared signal hypothesis to explain the interrelationship between gaze and emotion perception (e.g., Adams and Franklin 2009; Adams and Kleck 2003, 2005), suggesting that happiness and anger are preferably coupled with direct gaze, because both emotions are allegedly associated with approach oriented behavior, whereas fearful and sad expressions are coupled with averted gaze, as they are supposedly avoidance oriented. Others have argued that some emotions may be more accurately decoded when coupled with direct than with averted gaze because of greater behavioral relevance; for example an angry person with direct gaze might want to attack the observer (e.g., Sander et al. 2007). While these theories may explain certain aspects of the interrelationship between information of emotional expression and gaze direction, they fail to explain our present findings, suggesting that only happiness but not anger was associated with the feeling of being looked at (see also Lobmaier and Perrett 2011). Our present and previous findings rather 
suggest a distinction between positive and negative emotions and are more compatible with the idea that interpreting positive emotions as directed towards us and negative facial expressions as directed away may be beneficial for self-esteem (cf. Lobmaier and Perrett 2011; Lobmaier et al. 2008; Rimmele and Lobmaier 2011). Note that this interpretation is not in line with the findings of Adams and Franklin (2009). There are, however, several discrepancies between their study and ours. For example, Adams and Franklin (2009) used only two emotional expressions (angry and sad), and only two gaze angles (direct and averted). In contrast, our stimuli showed happy, angry, fearful and neutral expressions and were presented in 9 different horizontal viewing angles. Second, our paradigm required that participants indicate the exact location where they perceived the gaze to be directed at, rather than only deciding whether the face was looking at the observer or away. It will have to be the aim of future studies to fully elucidate the reasons for the different findings.

Our present data further show that the effect of expression was predominantly apparent in larger gaze angles, as reflected in the interaction of expression and rotation angle. In the smaller gaze angles the emotional expression modulated perceived gaze direction to a lesser extent, resulting in generally more accurate gaze perception in smaller angles. This is somewhat surprising, given that we previously found relatively large effects of emotion for faces looking only slightly to the left or right when deciding whether the face was making eye contact or not (Lobmaier and Perrett 2011; Lobmaier et al. 2008). While the reason for this inconsistency cannot be explained from the present data, we note that the paradigm and task differed between the two studies. Our previous studies adopted a two alternative forced choice task, while the participants in the present experiment could freely indicate the perceived gaze location. While gaze that is directed away from me by $6^{\circ}$ or more may clearly be perceived as not making eye contact, a dichotomous answer setting does not allow to determine other biases that may be pertinent to full gaze awareness. Conversely, a gaze that is almost meeting my eye may be perceived as making eye contact. But if asked to mark the exact location where this person is looking, the deviance from the veridical location may be small, thus resulting in small errors. Taken together, our findings show that the positivity bias in gaze perception is persistent in a task that has the advantage of offering a method that allows participants to freely indicate the perceived gaze location.

The accuracy of detecting where another person is looking was best for neutral faces and worst for fearful faces. The finding that the gaze perception of neutral faces was most accurate is in line with our predictions, because in these faces there were no interfering emotional cues.
However, the low accuracy in detecting where a fearful face is looking is somewhat surprising: to accurately detect the gaze direction of a person depicting fear could be seen as an adaptive mechanism to identify possible threats. Instead, our results suggest a tendency to interpret a potential threat to be located further away than it really is. Rather than demonstrating an adaptive strategy to locate sources of danger, our data are best explained by a general self-referential positivity bias: it may be beneficial for our self-esteem to interpret a potential threat as being far away from us, whereas self esteem is elevated by interpreting positive emotions as being directed towards ourselves. From an evolutionary perspective, this finding may mean that, rather than spending too much effort in detecting where exactly a threat may be, it may be sufficient to rapidly realize that there is a potential threat in the periphery and thus know in which direction not to run.

Previous studies have shown that human beings generally overestimate the gaze angle of other persons (Lobmaier et al. 2006; Schwaninger et al. 2005). In line with these findings, we found that the larger the rotation angle of the stimulus face, the more the gaze angle was overestimated. Looking at the emotions separately, this was true for angry, fearful and neutral emotions. In contrast, gaze angles of happy faces were more likely to be underestimated. Thus, it seems that the positivity bias is so powerful that it overwrites the general tendency to overestimate gaze angles.

We note that in this paper we use eye gaze direction as a general term referring to the direction somebody is looking without distinguishing between head direction and direction of the eyes relative to the head. We thus follow the rationale used in our previous work (e.g., Lobmaier and Perrett 2011). Such a definition might have lead to different results compared to if we had modified the direction of the eyes within a face. On the one hand, turning a head evidently also alters the visible proportion of the face, which may be important for emotion recognition. On the other hand, it seems more natural to also turn our head (not just our eyes) towards somebody to whom we want to communicate our emotional state. As such, frontal faces with modified eye direction might be ambiguous in that the emotion is directed towards the observer while the eyes are not. Critically, we found that even in larger angles the effect of emotion was present, in spite of the altered visible proportion of the face. In fact, the effect was even more pronounced in larger rotation angles.

Finally, recent studies have demonstrated that high anxious individuals show a greater sensitivity towards threat related stimuli, such as angry or fearful faces. For example, Tipples (2006) demonstrated that the gaze cueing effect towards fearful faces was correlated to participants' anxiety levels (see also Fox et al. 2007; Putman et al. 2006). In the present study we had not controlled for 
individual differences in anxiety levels, but on the background of the existing literature on anxiety as a personality trait we may speculate that the effects of emotion found in the present study would be even more pronounced, at least the effects for negative expressions. It will be the aim of future studies to implement this paradigm with a clinical population, where anxiety traits vary more than in student populations.

Taken together, we found a tendency to falsely interpret positive facial expressions as directed towards ourselves, whereas negative expressions are interpreted as directed away from us. This self-serving bias may be beneficial for self-esteem and is in line with previous studies reporting a self-referential positivity bias in gaze perception.

Acknowledgments Janek S. Lobmaier was supported by the Swiss National Science Foundation (grant number PZ00P1_121622/1). Matthias Hartmann and Andreas J. Volz were supported by a grant from the Pro*Doc program of the Swiss National Science Foundation awarded to FM (PDFMP1_127238).

\section{References}

Adams, R. B., Jr., \& Franklin, R. G., Jr. (2009). Influence of emotional expression on the processing of gaze direction. Motivation and Emotion, 33, 106-112.

Adams, R. B., Jr., \& Kleck, R. E. (2003). Perceived gaze direction and the processing of facial displays of emotion. Psychological Science, 14, 644-647.

Adams, R. B., Jr., \& Kleck, R. E. (2005). Effects of direct and averted gaze on the perception of facially communicated emotion. Emotion, 5, 3-11.

Ando, S. (2002). Luminance-induced shift in the apparent direction of gaze. Perception, 31(6), 657-674.

Borod, J. C., Santisch Haywood, C., \& Koff, E. (1997). Neuropsychological aspects of facial asymmetry during emotional expression: A review of the normal adult literature. Neuropsychology Review, 7, 41-60.

Darwin, C. (1872). The expression of the emotions in man and animals. London: John Murray.

Fox, E., Mathews, A., Calder, A. J., \& Yiend, J. (2007). Anxiety and sensitivity to gaze direction in emotionally expressive faces. Emotion, 7, 478-486.

Gale, C., \& Monk, A. F. (2000). Where am I looking? The accuracy of video-mediated gaze awareness. Perception \& Psychophysics, $62,586-595$.
Hietanen, J. K., \& Leppanen, J. M. (2003). Does facial expression affect attention orienting by gaze direction cues? Journal of Experimental Psychology: Human Perception and Performance, $29,1228-1243$.

Langton, S. R. (2000). The mutual influence of gaze and head orientation in the analysis of social attention direction. Quarterly Journal of Experimental Psychology A, 53, 825-845.

Langton, S. R., Watt, R. J., \& Bruce, I. I. (2000). Do the eyes have it? Cues to the direction of social attention. Trends in Cognitive Science, 4, 50-59.

Lee, K., Eskritt, M., Symons, L. A., \& Muir, D. (1998). Children's use of triadic eye gaze information for "mind reading". Developmental Psychology, 34, 525-539.

Lobmaier, J. S., \& Perrett, D. I. (2011). The world smiles at me: Selfreferential positivity bias when interpreting direction of attention. Cognition and Emotion, 25, 334-341.

Lobmaier, J. S., Fischer, M. H., \& Schwaninger, A. (2006). Objects capture perceived gaze direction. Experimental Psychology, 53, 117-122.

Lobmaier, J. S., Tiddeman, B., \& Perrett, D. I. (2008). Emotional expression modulates perceived gaze direction. Emotion, 8, 573-577.

Lobmaier, J. S., Mast, F. W., \& Hecht, H. (2010). For the mind's eye the world is two-dimensional. Psychonomics Bulletin and Review, 17, 36-40.

Perrett, D. I., Hietanen, J. K., Oram, M. W., \& Benson, P. J. (1992). Organization and functions of cells responsive to faces in the temporal cortex. Philosophical Transactions of the Royal Society London B, Biological Sciences, 335, 23-30.

Powell, W. R., \& Schirillo, J. A. (2009). Asymmetrical facial expressions in portraits and hemispheric laterality: A literature review. Laterality: Asymmetries of Body, Brain and Cognition, 14, 545-572.

Putman, P., Hermans, E., \& van Honk, J. (2006). Anxiety meets fear in perception of dynamic expressive gaze. Emotion, 6, 94-102.

Rimmele, U., \& Lobmaier, J. S. (2011). Stress increases the feeling of being looked at. Psychoneuroendocrinology. doi:10.1016/j.psy neuen.2011.1006.1013.

Sander, D., Grandjean, D., Kaiser, S., Wehrle, T., \& Scherer, K. R. (2007). Interaction effects of perceived gaze direction and dynamic facial expression: Evidence for appraisal theories of emotion. European Journal of Cognitive Psychology, 19, 470-480.

Schwaninger, A., Lobmaier, J. S., \& Fischer, M. H. (2005). The inversion effect on gaze perception reflects processing of component information. Experimental Brain Research, 167, 49-55.

Symons, L. A., Lee, K., Cedrone, C. C., \& Nishimura, M. (2004). What are you looking at? Acuity for triadic eye gaze. Journal of General Psychology, 131, 451-469.

Tipples, J. (2006). Fear and fearfulness potentiate automatic orienting to eye gaze. Cognition and Emotion, 20, 309-320. 